\title{
Satellite Communication Synchronization Based on Combining Multi-Differential Correlations in the High Doppler Channel
}

\author{
Jiancheng $L I U^{1,2}$, Saiyu $W A N G^{1}$, Zhisong $H A O^{1}$, Jingtao ZHANG \\ 1 The 54th Research Institute of China Electronics Technology Group Corporation, Zhongshan West Road No.589, 050003 \\ Shijiazhuang, China \\ 2 The Unit 31634 of PLA, Ganhaizi Street No.69, 611731 Kunming, China \\ ljcheng0807@hotmail.com.cn
}

Submitted July 25, 2020 / Accepted January 21, 2021

\begin{abstract}
In this paper, a pilot-aided synchronization method based on combining multi-differential correlations (MDCS) is proposed to overcome the obstacles in the synchronization of high-speed mobile satellite communication links. The proposed approach can optimally use the original synchronization pilot, and the existing transmitter would not need any modification. Additionally, the performance of proposed method is investigated on theoretical level, including the principle of mitigating the frequency offset deterioration and the derivation of closed synchronization probability expression. Finally, a set of simulations were operated to verify the MDCS performance, and the results proved that the MDCS significantly outperformed the existing approaches for large frequency offset and strong channel noise.
\end{abstract}

\section{Keywords}

Satellite communication, pilot-aided synchronization, multi-differential correlation, high Doppler channel, frequency offset

\section{Introduction}

Satellite communications is an important communication method due to its numerous advantages. Unfortunately, the large Doppler shift is inevitable for satellite communications. The synchronization between the transmitter and receiver is also a key step for satellite communication system, and the impairment of this synchronization caused by Doppler shift could deteriorate the transmission. Therefore, besides carrier synchronization [1], the time synchronization with a large frequency offset is also important for establishing a stable and reliable satellite communication link. Currently, the time synchronization methods can be categorized into a high-precision time service [2] and pilot-aided correlation (PAC) [3]. The first method is bound to the time service equipment because of its requirements of a unique high-precision clock source, such as global positioning system or Galileo and BeiDou systems. The PAC has high accuracy and strong anti-noise ability; however, this method is very sensitive to the frequency offset (FO). In [3], the IEEE $802.11 \mathrm{p}$ standard and orthogonal frequency-division multiplexing (OFDM) was used, and the synchronization pilot was equally divided into two conjugated parts to enhance the synchronization performance to some extent. However, this method failed to synchronize when the FO period was less than the pilot length and was not appropriate for satellite communications [5]. The training pilot of Zadoff-Chu (ZC) sequence was adopted in [6,7], called ZC sequence correlation synchronization (ZCCS) method. On the other hand, some other synchronization methods and analysis were studied in [8-10].

Besides land mobile communication, the new synchronization pilot consisting of two conjugate ZC sequences was applied to satellite communication in [11,12], which could tolerate the FO with non-integer multiples of the pilot time period. Nonetheless, the synchronization method designed for OFDM was rarely used in satellite communications. In [13], the authors improved the structure and position of synchronization pilot for the low earth orbit (LEO) satellite communication system and adopted the method used in [11] to reject the FO. In [14], the PAC synchronization method based on the special structure was proposed for the second generation digital video broadcasting transmission system, which took advantage of the conjugate symmetry of the pilot sequence. In [15], the differentially coherent detectors of the pseudorandom noise (PN) code was proposed for the codedivision multiple-access (CDMA) systems, and the closed expressions of the detection and false alarm probabilities were derived. In [16], the differential post-detection integration techniques were investigated, and the missed detection and false alarm probabilities of various differential schemes were theoretically analyzed. Among the correlation methods, the differential post integration is robust to the FO, but this 
method should be further rectified to be suitable for satellite communication links with high FO and strong noise. Thus, we build the synchronization pilot of $\mathrm{PN}$ sequence with the same characters as modulation symbols, and the received signal is multiplied by the conjugate of local pilot. The product is operated on $N$ different delays, and then each delayed sequence is executed with difference computation respectively. After that, the results of $N$ difference computation are equally combined to obtain the correlation peak of synchronization. The proposed method can both resist the FO and suppress the noise to ensure the reliable and accurate time synchronization.

The remaining sections are organized as follows. In Section 2, the model of non-differential cross-correlation synchronization (NDCS) and common differential correlation synchronization (CDCS) methods are analyzed, and the model of proposed MDCS method is built and elaborated. The robustness to FO is investigated for different synchronization methods, and the closed expressions of synchronization probability and peak-to-noise ratio (PNR) are presented in Section 3, for performance comparisons of models. The simulation under various conditions and analytical results are discussed in Sec 4. Finally, conclusions are given in Sec. 5.

\section{Synchronization Model for MDCS}

The popular synchronization method is PAC method, which can quickly determine the start moment of the received signal to ensure reliable transmission. Finally, we introduce the MDCS method based on PAC.

\subsection{Principles of NDCS}

The PAC synchronization inserts the designed pilot in front of the information symbols, as shown in Fig. 1. The pilot must have a superior autocorrelation property that is conducive to detecting the cross-correlation peak between the received signal and local pilot.

Let $\boldsymbol{p}_{\mathrm{L}}$ represent the pilot symbol array that is formed as $\boldsymbol{p}_{\mathrm{L}}=\left[p_{\mathrm{L}}^{1} p_{\mathrm{L}}^{2} \cdots p_{\mathrm{L}}^{L}\right]$, where the subscript $L$ is the length of pilot; $p_{\mathrm{L}}^{n}=p_{L, \mathrm{i}}^{n}+j p_{L, \mathrm{q}}^{n}$ is normalized to satisfy $p_{\mathrm{L}}^{n *} \cdot p_{\mathrm{L}}^{n}=1$, where the asterisk denotes complex conjugate. The transmitted symbol array $\boldsymbol{s}$ consists of the pilot symbol array $\boldsymbol{p}_{\mathrm{L}}$ and the information symbol array $\boldsymbol{x}_{n}(n=1,2, \cdots)$, that is, $\boldsymbol{s}=\left[p_{\mathrm{L}}^{1} p_{\mathrm{L}}^{2} \cdots p_{\mathrm{L}}^{L} x_{1} x_{2} \cdots\right]$. The signal received as the output of the transmission channel can be written as

$$
r(n)=\sum_{m=1}^{M} h(m) s(n-m) \mathrm{e}^{\mathrm{j} \omega(n)}+\varepsilon(n)
$$

where $\varepsilon(n)$ is the additive noise in the channel; $h(m)$ is the coefficient of channel response, $M$ is the order of channel response, and $\mathrm{e}^{\mathrm{j} \omega(n)}$ represents the FO generated by the transmission channel.

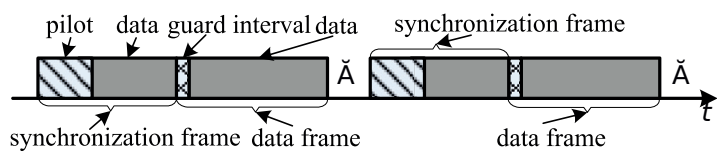

Fig. 1. Data structure of PAC synchronization.

At the receiver, the correlation between $r(n)$ and local pilot $p_{\mathrm{L}}^{l}$ is calculated. If the correlation result is defined as $Y(n)$, the output value of NDCS can be deduced as follows:

$$
R_{\mathrm{NDC}}(n)=|Y(n)|=\left|\sum_{l=0}^{L-1}\left[p_{\mathrm{L}}^{(L-l) *} \cdot r(n-l)\right]\right| .
$$

From the above, we know that $R_{\mathrm{NDC}}(n)$ can achieve the max value only if $r(n)$ matches $p_{\mathrm{L}}^{l}$.

\subsection{Synchronization Based on MDCS}

Figure 2 shows the process of synchronization (which is unlike NDCS) obtained by MDCS. In Fig. 2, where the $\mathrm{Z}^{-1}$ signifies the delay tap of one symbol period, and $|*|$ denotes the absolute value. The process is as follows. First, the product of received signal $r(n)$ and conjugate of local pilot $p_{\mathrm{L}}^{l}$ are computed. Second, the output sequence $X(n)$ is respectively passed by $N$ types of fixed delays, and the conjugate products of $N$ delay types and $X(n)$ are obtained, which are marked as $\bar{X}_{i}(n), i=1,2, \cdots, N$. This step is a multidifferential operation. Third, the correlation output of each path is separately calculated. Then the $N$ correlation values are equally combined to achieve the differential correlation output $\bar{R}(n)$. Finally, the correlation output of this method $R_{\mathrm{MDC}}(n)$ is obtained by summing $\bar{R}(n)$ and $R_{\mathrm{MDC}}(n)$ (which are the outputs of NDCS) with the weighting factors $\mu_{1}$ and $\mu_{2}$, respectively.

The mathematical description of the synchronization process is as follows:

$$
\begin{aligned}
& X(n-L+i)=\left(p_{\mathrm{L}}^{i}\right)^{*} \cdot r(n-L+i), \quad i=1, \cdots, L, \\
& \bar{X}_{i}(n)=\operatorname{conj}[X(n)] \cdot X(n-i), \quad i=1, \cdots, N, \\
& \left\{\begin{array}{l}
Y(n)=\sum_{l=0}^{L-1} X(n-l) \\
\bar{Y}_{i}(n)=\sum_{l=0}^{L-i-1} \bar{X}_{i}(n-l), i=1,2, \cdots, N,
\end{array}\right. \\
& \left\{\begin{array}{l}
\bar{R}(n)=\frac{1}{N} \sum_{k=1}^{N} \mid \bar{Y}_{k}(n) \\
R_{\mathrm{NDC}}(n)=|Y(n)| .
\end{array}\right.
\end{aligned}
$$

Finally, we introduced the weighting factors and ensured that $\mu_{1}$ and $\mu_{2}$ satisfy the condition $\mu_{1}+\mu_{2}=1$. Then, we can achieve the final correlation output $R_{\mathrm{MDC}}(n)$ to detect the following synchronization:

$$
R_{\mathrm{MDC}}(n)=\mu_{1} \cdot \bar{R}(n)+\mu_{2} \cdot R_{\mathrm{NDC}}(n)
$$




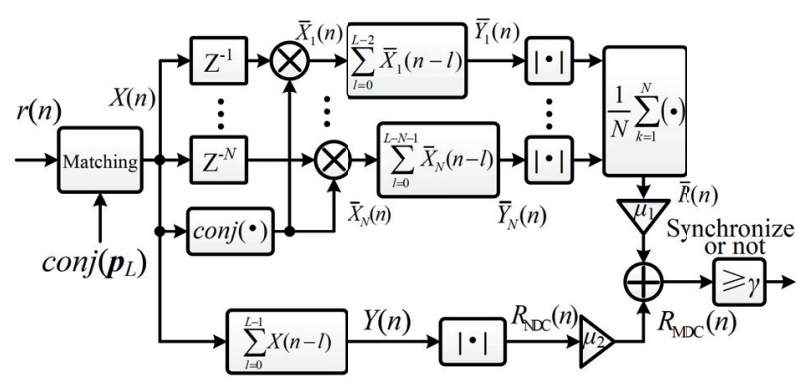

Fig. 2. Structure of MDCS.

Synchronization detection is used to compare $R_{\mathrm{MDC}}(n)$ with the decision threshold $\gamma$; the moment of $R_{\mathrm{MDC}}(n)$ greater than $\gamma$ is the synchronization flag. The pilot of proposed method has the same signal characters as the binary/quadrature phase-shift key (BPSK/QPSK) modulated signals applied in satellite communications; these characters can decrease the detect ability of the pilot.

\section{Performance Analysis}

\subsection{Influence of Doppler Shift}

Satellite transmission occurs in the line of sight; therefore, the impairment of the transmitted signal is primarily caused by the path loss, environment noise, dynamic property, and imperfect components [17], which would mainly result in signal decays and FOs. Therefore, the channel response $h(m)$ given in (1) can be simplified as the signal amplitude attenuation. Then, the received signal $r(n)$ can be further expressed as

$$
r(n)=\beta p_{\mathrm{L}}^{n} \mathrm{e}^{\mathrm{j}\left(2 \pi f_{\Delta} \frac{n}{f_{\mathrm{s}}}+\theta_{0}\right)}+\varepsilon(n)
$$

where $f_{\mathrm{s}}=1 / T_{\mathrm{S}}$ is sample rate that is equivalent to symbol rate; $\beta$ is attenuation factor, $f_{\Delta}$ and $\theta_{0}$ are the frequency and phase offset values, respectively, caused by dynamic channel and imperfect components, and $n$ can be $1, \cdots, L$.

For the NDCS method [3, 13], the correlation value $R_{\mathrm{NDC}}(L)$ between received signal and local pilot can be calculated by using (2) and (8) as follows:

$$
\begin{gathered}
R_{\mathrm{NDC}}(L)=\left|\beta \mathrm{e}^{\mathrm{j}\left[2 \pi f_{\Delta} \frac{L}{\mathrm{f}_{\mathrm{s}}}+\theta_{0}\right]} \cdot \frac{\sin \left(\pi f_{\Delta} L / f_{\mathrm{s}}\right)}{\sin \left(\pi f_{\Delta} / f_{\mathrm{s}}\right)}+\varepsilon_{\mathrm{L}}\right|, \\
\varepsilon_{\mathrm{L}}=\sum_{l=0}^{L-1}\left\{\left(p_{\mathrm{L}}^{L-l}\right)^{*} \cdot \varepsilon(L-l)\right\}=\varepsilon_{\mathrm{L}, \mathrm{i}}+\mathrm{j} \varepsilon_{\mathrm{L}, \mathrm{q}} .
\end{gathered}
$$

From (9), we know that the correlation peak value depends mainly on the FO $f_{\Delta}$ besides channel noise $\varepsilon_{\mathrm{L}}$. Commonly, the FO $f_{\Delta}$ would be much smaller than the sample rate $f_{\mathrm{s}}$. Further analysis shows that the impact of FO $f_{\Delta}$ on the correlation peak $R_{\mathrm{NDC}}(L)$ would be most serious when $f_{\Delta}$ and $f_{\mathrm{s}}$ satisfy $f_{\Delta} L / f_{\mathrm{s}}=M$, in which $M$ is an integer.

Given the proposed MDCS method, when $n=L$, the $R_{\mathrm{NDC}}(L)$ computation is the same as that in (10). Then, we examine the relationship between $\bar{R}(L)$ and $f_{\Delta}$.
First, $\bar{X}_{k}(n)$ in (4) can be calculated as follows:

$$
\begin{aligned}
\left.\bar{X}_{k}(n)\right|_{k=1, \cdots, N} & =\left(\beta \mathrm{e}^{-\mathrm{j}\left[2 \pi f_{\Delta} \frac{n}{f_{\mathrm{s}}}+\theta_{0}\right]}+p_{\mathrm{L}}^{n} \cdot \varepsilon^{*}(n)\right) . \\
& \left(\beta \mathrm{e}^{\mathrm{j}\left[2 \pi f_{\Delta} \frac{n-k}{f_{\mathrm{s}}}+\theta_{0}\right]}+\left(p_{\mathrm{L}}^{n-k}\right)^{*} \cdot \varepsilon(n-k)\right)
\end{aligned}
$$

By denoting

$$
\left\{\begin{array}{l}
\varepsilon_{k, 1}(n)=\beta p_{\mathrm{L}}^{n} \varepsilon^{*}(n) \mathrm{e}^{\mathrm{j}\left(2 \pi f_{\Delta} \frac{n-k}{f_{\mathrm{s}}}+\theta_{0}\right)} \\
\varepsilon_{k, 2}(n)=\beta\left(p_{\mathrm{L}}^{n-k}\right)^{*} \cdot \varepsilon(n-k) \mathrm{e}^{-\mathrm{j}\left(2 \pi f_{\Delta} \frac{n}{f_{\mathrm{s}}}+\theta_{0}\right)} \\
\varepsilon_{k, 3}(n)=p_{\mathrm{L}}^{n} \cdot\left(p_{\mathrm{L}}^{n-k}\right)^{*} \cdot \varepsilon^{*}(n) \cdot \varepsilon(n-k) \\
\bar{\varepsilon}_{L, k}=\sum_{l=0}^{L-k-1}\left[\varepsilon_{k, 1}(L-l)+\varepsilon_{k, 2}(L-l)+\varepsilon_{k, 3}(L-l)\right] .
\end{array}\right.
$$

From (11), the differential accumulation output $\bar{Y}_{k}(L)$ of the matching moment is expressed as follows:

$$
\bar{Y}_{k}(L)=\sum_{l=0}^{L-k-1}\left(\beta^{2} \mathrm{e}^{-\mathrm{j} 2 \pi f_{\Delta} \frac{k}{f_{\mathrm{s}}}}\right)+\bar{\varepsilon}_{L, k} .
$$

Let $\bar{R}_{k}(n)=\left|\bar{Y}_{k}(n)\right|$, that is, $\bar{R}_{k}(n)=\sqrt{\left|\bar{Y}_{k, \mathrm{i}}(n)\right|^{2}+\left|\bar{Y}_{k, \mathrm{q}}(n)\right|^{2}}$, which is the output of the $\mathrm{k} t h$ differential correlation. Here, $\bar{Y}_{k, \mathrm{i}}(n)$ and $\bar{Y}_{k, \mathrm{q}}(n)$ are the real and imaginary components of $\bar{Y}_{k}(n)$, respectively. Then, the $\bar{R}(L)$ of MDCS at the matching moment can be written as follows:

$$
\bar{R}(L)=\frac{1}{N} \sum_{k=1}^{N}\left|(L-k) \beta^{2} \mathrm{e}^{-\mathrm{j} 2 \pi f_{\Delta} \frac{k}{f_{\mathrm{s}}}}+\bar{\varepsilon}_{L, k}\right| .
$$

For analyzing the FO impact, we can ignore channel noise. Then, the peak value $\bar{R}(L)$ [16] can be simplified as:

$$
\bar{R}(L)=\frac{2 L-N-1}{2} \beta^{2} .
$$

Usually, the $L$ is much larger than $N$. Hence, the correlation peak value of MDCS is not impacted by the FO. Finally, we can achieve the $R_{\mathrm{MDC}}(L)$ of MDCS without channel noise, and it is written as follows:

$$
R_{\mathrm{MDC}}(L)=\mu_{1} \beta^{2} \frac{2 L-N-1}{2}+\mu_{2} \beta\left|\frac{\sin \left(\pi f_{\Delta} L / f_{\mathrm{s}}\right)}{\sin \left(\pi f_{\Delta} / f_{\mathrm{s}}\right)}\right| .
$$

In addition, the values of weighting factors $\mu_{1}$ and $\mu_{2}$ were decided by the channel condition. In other words, if the channel is provided with a large FO due to the Doppler, two weighting factors should be restrained with $\mu_{1} \gg \mu_{2}$. In this case, the $R_{\mathrm{MDC}}(L)$ would be primarily dominated by $\bar{R}(L)$ rather than $f_{\Delta}$. The proposed method can strongly prevent the FO and will successfully build synchronization in the high Doppler channel.

\subsection{Analysis of Synchronization Probabilities}

By investigating the rejecting FO in subsection 3.1, we will examine the synchronization probabilities of the proposed and existing methods $[3,13,16]$. In the Doppler channel with additive white noise, $\varepsilon(n)=\varepsilon_{\mathrm{i}}(n)+\mathrm{j} \varepsilon_{\mathrm{q}}(n)$ is 
assumed as the expression of complex noise. The real and imaginary parts of the noise are subject to the same normal distribution and are independent of each other. The mean value and standard deviation are respectively assumed to be 0 and $\sqrt{2} \sigma_{\varepsilon} / 2$, that is $E\left[\varepsilon(n) \varepsilon^{*}(n)\right]=\sigma_{\varepsilon}^{2}$.

\section{(a) Synchronization Probability of Existing Method}

When the received signal is matched with local pilot in existing method, $Y(L)$ can be written as follows:

$$
Y(L)=\beta \mathrm{e}^{\mathrm{j}\left(2 \pi f_{\Delta} \frac{L}{f_{\mathrm{s}}}+\theta_{0}\right)} \cdot \frac{\sin \left(\pi f_{\Delta} L / f_{\mathrm{s}}\right)}{\sin \left(\pi f_{\Delta} / f_{\mathrm{s}}\right)}+\varepsilon_{\mathrm{L}} .
$$

Every $p_{\mathrm{L}}^{n}$ has the same modulus (equal to 1) and presence probability, the probability distribution of real component $\varepsilon_{L, \mathrm{i}}$ and imaginary component $\varepsilon_{L, \mathrm{q}}$ for the rectified noise $\varepsilon_{\mathrm{L}}$ is also normal. Therefore, we can let $\omega^{\prime}=2 \pi f_{\Delta} L / f_{\mathrm{s}}+\theta_{0}$. Then, $Y(L)$ would be further expressed as follows:

$$
Y(L)=\beta \frac{\sin (L \varphi)}{\sin (\varphi)}\left[\cos \left(\omega^{\prime}\right)+\mathrm{j} \sin \left(\omega^{\prime}\right)\right]+\varepsilon_{L, \mathrm{i}}+\mathrm{j} \varepsilon_{L, \mathrm{q}}
$$

From the above expression, we can know that real component $Y_{\mathrm{i}}(L)$ and imaginary component $Y_{\mathrm{i}}(L)$ of $Y(L)$ are also subject to normal distribution; their mean value and deviation will be written as follows:

$$
\begin{aligned}
& \left\{\begin{array}{l}
u_{\mathrm{i}}=\beta \frac{\sin (L \varphi)}{\sin (\varphi)} \cos \left(\omega^{\prime}\right) \\
u_{\mathrm{q}}=\beta \frac{\sin (L \varphi)}{\sin (\varphi)} \sin \left(\omega^{\prime}\right),
\end{array}\right. \\
& \left\{\begin{array}{l}
\sigma_{\mathrm{i}}^{2}=L \sigma_{\varepsilon}^{2} / 2 \\
\sigma_{\mathrm{q}}^{2}=L \sigma_{\varepsilon}^{2} / 2 .
\end{array}\right.
\end{aligned}
$$

Moreover, the correlation peak $R_{\mathrm{NDC}}(L)$ is equal to the modulus of $Y(L)$ :

$$
R_{\mathrm{NDC}}(L)=\sqrt{\left|Y_{\mathrm{i}}(L)\right|^{2}+\left|Y_{\mathrm{q}}(L)\right|^{2}} .
$$

From (19-21), it is obvious that the $R_{\mathrm{NDC}}(L)$ is a random variable of Ricean distribution [18], and its probability density function can be expressed as

$$
\begin{aligned}
f_{R_{\mathrm{NDC}}}(z)= & \frac{2 z}{L \sigma_{\varepsilon}^{2}} \exp \left[\frac{-1}{L \sigma_{\varepsilon}^{2}}\left(z^{2}+\beta^{2} \frac{\sin ^{2}(L \varphi)}{\sin ^{2}(\varphi)}\right)\right] . \\
& I_{0}\left(\frac{2 z \beta \sin (L \varphi)}{L \sigma_{\varepsilon}^{2} \sin (\varphi)}\right)
\end{aligned}
$$

where $I_{0}(\cdot)$ is the rectified Bessel function of the first kind with the 0 order.

Based on the probability density function presented in (22), we can easily achieve the synchronization probability of the existing non-differential method as follows:

$$
P_{\mathrm{NDC}}=\int_{\gamma}^{+\infty} f_{R_{\mathrm{NDC}}}(z) \mathrm{d} z
$$

\section{(b) Synchronization Probability of Proposed Method}

From the expression (7), we can know that the correlation peak value of proposed method would depend on $R_{\mathrm{NDC}}(L)$ and $\bar{R}(L)$. The probability distribution of $R_{\mathrm{NDC}}(L)$ has been studied in previous subsection, so we would deduce the probability distribution of $\bar{R}(L)$ in this subsection.

Based on (3-5), the mathematical relationship between the differential path output $\bar{Y}_{k}(L)$ and the matched output sequence $X(n)$ can be written as follows:

$$
\bar{Y}_{k}(L)=\sum_{l=0}^{L-k-1}\{\operatorname{conj}[X(L-l)] \cdot X(L-l-k)\} .
$$

We concluded that the matched output $X(n)$ is also a normal distribution because $r(n)$ is a normal distribution, and the local pilot sequence $p_{\mathrm{L}}^{n}$ has a constant modulus and random property. Consequently, all the elements of $\bar{X}_{k}(n)$ are independent and identically distributed. According to central limit theorem [14], when the length $L$ of pilot is sufficiently large, $\bar{Y}_{k}(L)$ can be approximated as a complex normal distribution. Therefore, we can evaluate the mean value and deviation of $\bar{Y}_{k}(L)$ via the mean value and deviation of $\bar{X}_{k}(n)$ and further obtain the probability distribution of $\bar{Y}_{k}(L)$. From (13), $\bar{X}_{k}(n)$ can be written as

$$
\bar{X}_{k}(n)=\beta^{2} \mathrm{e}^{-\mathrm{j} 2 \pi f_{\Delta} \frac{k}{f_{\mathrm{s}}}}+\varepsilon_{k, 1}(n)+\varepsilon_{k, 2}(n)+\varepsilon_{k, 1}(n),
$$

where $k=1, \cdots, N$. Its mean value and deviation are respectively computed as follows:

$$
\left\{\begin{array}{l}
u_{\bar{X}_{k}}=\beta^{2} \mathrm{e}^{-\mathrm{j} 2 \pi f_{\Delta} \frac{k}{f_{\mathrm{s}}}} \\
\sigma_{\bar{X}_{k}}^{2}=2 \beta^{2} \sigma_{\varepsilon}^{2}+\sigma_{\varepsilon}^{4} .
\end{array}\right.
$$

By using the above analysis, the mean value and deviation of $\bar{Y}_{k}(L)$ can be obtained as follows:

$$
\left\{\begin{array}{l}
u_{\bar{Y}_{k}}=(L-k) \beta^{2} \mathrm{e}^{-\mathrm{j} 2 \pi f_{\Delta} \frac{k}{f_{s}}} \\
\sigma_{\bar{Y}_{k}}^{2}=(L-k)\left(2 \beta^{2} \sigma_{\varepsilon}^{2}+\sigma_{\varepsilon}^{4}\right) .
\end{array}\right.
$$

Consequently, $\bar{R}_{k}(L)$ is subject to the Ricean distribution because $\bar{R}_{k}(L)=\sqrt{\left|\bar{Y}_{k, \mathrm{i}}(L)\right|^{2}+\left|\bar{Y}_{k, \mathrm{q}}(L)\right|^{2}}$, and its probability density function can be written as follows:

$$
f_{\bar{R}_{k}}(z)=\frac{2 z}{\sigma_{\bar{Y}_{k}}^{2}} \exp \left[\frac{-1}{\sigma_{\bar{Y}_{k}}^{2}}\left(z^{2}+\left|u_{\bar{Y}_{k}}\right|^{2}\right)\right] I_{0}\left(2 \frac{z\left|u_{\bar{Y}_{k}}\right|}{\sigma_{\bar{Y}_{k}}^{2}}\right) \text {. }
$$

Therefore, the probability distribution $F_{\bar{R}}$ of $\bar{R}(L)$ can be given as

$$
F_{\bar{R}}(s<x)=\oint_{s<x}\left[\prod_{k=0}^{N-1} f_{\bar{R}_{k}}\left(z_{k}\right)\right] \mathrm{d} z_{0} \cdots \mathrm{d} z_{N-1},
$$

where $s=\frac{1}{N} \sum_{k=0}^{N-1} z_{k}$. From (22) and (29), we can derive the probability $P_{\mathrm{R}}$ of the correlation peak $R_{\mathrm{MDC}}(L)$, which is larger than the threshold $\gamma$ expressed as follows:

$$
P_{R_{\mathrm{MDC}}}=\int_{0}^{+\infty}\left[1-F_{\bar{R}}\left(\frac{\gamma-\mu_{2} z}{\mu_{1}}\right)\right] f_{R_{\mathrm{NDC}}}(z) \mathrm{d} z .
$$


When the probability density functions $f_{\bar{R}_{k}}(z)$ and $f_{R_{\mathrm{NDC}}}(z)$ are fixed, the synchronization probability of proposed method is primarily dominated by weighting factors $\mu_{1}$ and $\mu_{2}$, and the ratio of two weighting factors can change the impact of $\bar{R}(L)$ and $R_{\mathrm{NDC}}(L)$ on $R_{\mathrm{MDC}}(L)$. If the FO in channel is large, we would approximate the weighting factor $\mu_{1}$ to 1 and the weighting factor $\mu_{2}$ to 0 . On the contrary, we would approximate the weighting factor $\mu_{1}$ to 0 and the weighting factor $\mu_{2}$ to 1 for a small FO. In addition, if we are not able to previously evaluate the channel condition, the weighting factors $\mu_{1}$ and $\mu_{2}$ should be equally set to 0.5 .

\subsection{Performance of Anti-Noise}

In Doppler channel, in addition to rejecting FO, the ability of anti-noise is also crucial. Therefore, we will investigate the anti-noise performance of proposed method by comparing it with the NDCS and CDCS methods. Analysis of the anti-noise performance is based on PNR. The PNR values of three methods are $\mathrm{PNR}_{\mathrm{NDC}}, \mathrm{PNR}_{\mathrm{CDC}}$ and $\mathrm{PNR}_{\mathrm{MDC}}$.

Based on (18) and (21), the pure correlation $\mathrm{PNR}_{\mathrm{NDC}}$ of NDCS method is written as follows:

$$
\mathrm{PNR}_{\mathrm{NDC}}=20 \cdot \log _{10}\left[\sqrt{\frac{\beta^{2} \sin ^{2}(L \varphi)}{L \sigma_{\varepsilon}^{2} \sin ^{2}(\varphi)}}\right] .
$$

Given the CDCS method, it can be regarded as the proposed method MDCS with $N=1$. Then, the pure correlation $\mathrm{PNR}_{\mathrm{CDC}}$ of the CDCS method is written as follows:

$$
\mathrm{PNR}_{\mathrm{CDC}}=20 \cdot \log _{10}\left[\sqrt{\frac{(L-1) \beta^{4}}{2 \beta^{2} \sigma_{\varepsilon}^{2}+\sigma_{\varepsilon}^{4}}}\right] .
$$

Based on (32), we can respectively derive the PNRs for $N$ differential paths of MDCS. From (6) and (7), we know that $\mathrm{PNR}_{\mathrm{MDC}}$ is related to $\mathrm{PNR}_{\mathrm{NDC}}$ and $\mathrm{PNR}_{\mathrm{CDC}}$. The $\mathrm{PNR}_{\mathrm{MDC}}$ can be achieved as follows:

$$
\mathrm{PNR}_{\mathrm{MDC}}=20 \cdot \log _{10}\left[\begin{array}{l}
\mu_{1} \sqrt{\frac{N(2 L-N-1) \beta^{4}}{2\left(2 \beta^{2} \sigma_{\varepsilon}^{2}+\sigma_{\varepsilon}^{4}\right)}} \\
+\mu_{2} \sqrt{\frac{\beta^{2} \sin ^{2}(L \varphi)}{L \sigma_{\varepsilon}^{2} \sin ^{2}(\varphi)}}
\end{array}\right] .
$$

By comparing (32) with (323, we can see that even if the weighting factor $\mu_{2}$ is equal to $0, \mathrm{PNR}_{\mathrm{MDC}}$ of the proposed method is still larger than $\mathrm{PNR}_{\mathrm{CDC}}$ of the CDCS method because $L \gg N$. This proves that the proposed MDCS method is robust to noise.

\section{Simulation Results}

In this section, to demonstrate the efficiency and robustness to FO and channel noise of MDCS, we simulate the proposed method and compare its results with the existing methods, including the common NDCS method, the CDCS method of [16], and the ZCCS method of [11]. The simulations using the QPSK modulation are popular in satellite communications, and the symbol rate is assumed to be 10.24 Msps. The highest speed for the rapidly moving target can be close to $30 \mathrm{Mach}$, and the target may move opposite to satellite in low-earth and middle-earth orbits. Therefore, if the carrier frequency is in the Ka frequency band, the $f_{\Delta}$ caused by the Doppler effect should be assumed from $100 \mathrm{~Hz}$ to $4 \mathrm{MHz}$. In addition, the pilot of the proposed method is constructed by the modulated $\mathrm{m}$ sequence, and the pilot length $L$ is set to 1023 for all methods. The received signal is normalized, and the threshold $\gamma$ of the correlation peak detection is $L / 6$. All results in this section have been obtained by the software MATLAB with averaging the Monte Carlo simulation 1000 times.

\subsection{Comparison of Different Methods}

First, we simulate the synchronization probabilities of four methods with the FOs of $500 \mathrm{~Hz}, 10 \mathrm{kHz}, 200 \mathrm{kHz}$ and $4 \mathrm{MHz}$. It is assumed that the channel information is unknown; therefore, we let $\mu_{1}=\mu_{2}=0.5$ for proposed method, and specify the number $N$ of the differential path as 8 . The simulation results are shown in Fig. 3.

From the probability curves shown in Fig. 3, we can achieve the $E_{\mathrm{S}} / N_{0}$ values of the four methods for a synchronization probability larger than $99 \%$, as shown in Tab. 1 .

From Fig. 3 and Tab.1, it is obvious that when the FO is $500 \mathrm{~Hz}$, the $E_{\mathrm{S}} / N_{0}$ value of proposed MDCS method would be $1.5 \mathrm{~dB}$ less than the NDCS method but $6 \mathrm{~dB}$ greater than the CDCS method. While the FO increases to $10 \mathrm{kHz}, 200 \mathrm{kHz}$ and $4 \mathrm{MHz}$, the NDCS method cannot be successfully synchronized, and the $E_{\mathrm{s}} / N_{0}$ values of the CDCS method still remain at approximately $-6 \mathrm{~dB}$. However, the values of proposed MDCS method are approximately $-7.5 \mathrm{~dB}$. Moreover, the $E_{\mathrm{S}} / N_{0}$ values of the ZCCS method show extreme differentiation between the varying FOs, and even this method cannot achieve the synchronization probability of $99 \%$ for the FO of $10 \mathrm{kHz}$ and $4 \mathrm{MHz}$. The results prove that the NDCS and the proposed MDCS method are remarkably superior to the CDCS and ZCCS methods for small FOs. When the FO is not less than $10 \mathrm{kHz}$, the NDCS method is not able to synchronize; conversely, the CDCS and the proposed MDCS methods have a constant synchronization property, that is, the two methods are barely impacted by the varying FO.

\begin{tabular}{|c|c|c|c|c|}
\hline FO & NDCS & CDCS & ZCCS & MDCS \\
\hline $500 \mathrm{~Hz}$ & $-13.5 \mathrm{~dB}$ & $-6 \mathrm{~dB}$ & $-10 \mathrm{~dB}$ & $-12 \mathrm{~dB}$ \\
\hline $10 \mathrm{kHz}$ & - & $-6 \mathrm{~dB}$ & - & $-7.5 \mathrm{~dB}$ \\
\hline $200 \mathrm{kHz}$ & - & $-6 \mathrm{~dB}$ & $-10 \mathrm{~dB}$ & $-7.5 \mathrm{~dB}$ \\
\hline $4 \mathrm{MHz}$ & - & $-6 \mathrm{~dB}$ & $-2 \mathrm{~dB}$ & $-7.5 \mathrm{~dB}$ \\
\hline
\end{tabular}

Tab. 1. $E_{\mathrm{S}} / N_{0}$ values of different methods for synchronization probability larger than $99 \%$.

Given the ZCCS method, the synchronization probability with high $E_{\mathrm{S}} / N_{0}$ would severely fluctuate for different FO values. This happens because the fixed detection threshold would give rise to a false alarm for a high $E_{\mathrm{S}} / N_{0}$ in the ZCCS 

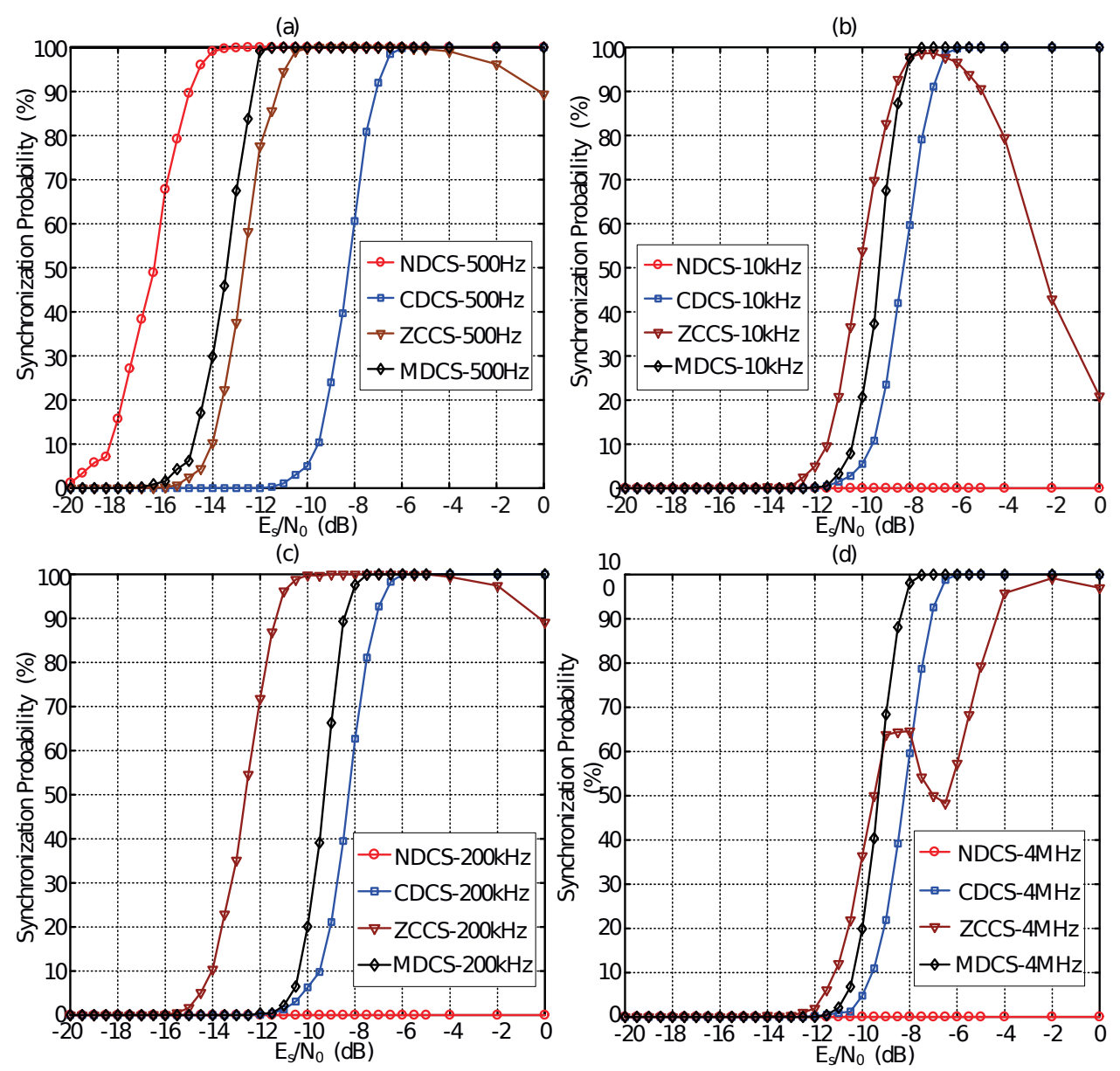

Fig. 3. Synchronization probabilities of four methods, the weighting factors of proposed method are $\mu_{1}=\mu_{2}=0.5$, and differential path number $N$ is 8, the propagation channel is assumed as AWGN channel: (a) FO of $500 \mathrm{~Hz}$, (b) FO of $10 \mathrm{kHz}$, (c) FO of $200 \mathrm{kHz}$, (d) FO of $4 \mathrm{MHz}$.

method. In conclusion, when the weighting factors are equal, the proposed MDCS method can both have a strong ability of anti-noise for small FOs and can successfully achieve synchronization for large FOs. If we can rectify the weighting factor values by evaluating the channel information, the synchronization performance of the proposed MDCS method can be further improved.

\subsection{Results of Different Weighting Factors}

In this subsection, the influence of the weighting factor on the synchronization performance of the MDCS method is analyzed using simulation results, which are compared with the CDCS and NDCS methods. To enhance the ability of resisting the FO, the weighting factors should be restrained with $\mu_{1} \geq \mu_{2}$. The FO in the simulation is set to the two values of $200 \mathrm{kHz}$ and $4 \mathrm{MHz}$, and the weighting factors are assumed to be the four values $\mu_{1}=0.75, \mu_{2}=0.25 ; \mu_{1}=0.9, \mu_{2}=0.1$; $\mu_{1}=0.99, \mu_{2}=0.01 ; \mu_{1}=0.999, \mu_{2}=0.001$. The simulation results of the FO of $200 \mathrm{kHz}$ and $4 \mathrm{MHz}$ are shown in Fig. 4(a) and Fig. 4(b), respectively. Combining Fig. 4(a) and (b), we can draw a conclusion that the synchronization probability curves of MDCS method are almost the same for the two FO values of $200 \mathrm{kHz}$ and $4 \mathrm{MHz}$, which demon- strates that the synchronization performance of MDCS was consistent for different FO values. In addition, it is clear that the weighting factor value can influence the synchronization performance. The $E_{\mathrm{s}} / N_{0}$ value of a fixed synchronization probability would decrease when the weighting factor $\mu_{1}$ is increased, and the improvement of the synchronization performance is no longer evident when $\mu_{1}$ larger than 0.99 . In other words, the synchronization probability with weighting factors $\mu_{1}=1, \mu_{2}=0$ is almost equal to the value of weighting factors $\mu_{1}=0.99, \mu_{2}=0.01$. Therefore, from Fig. 4 we know that the weighting factor $\mu_{1}$ should be 0.99 for the channel with a large FO.

\subsection{Influence of the Path Number $N$}

In this subsection, we investigate the influence of the path number $N$ on the synchronization performance, and the number $N$ is assumed to have the five values of $2,4,8$, 12 and 16. The simulations are operated via the weighting factors $\mu_{1}=0.99, \mu_{2}=0.01$ and FO values of $200 \mathrm{kHz}$, $4 \mathrm{MHz}$. The simulation results are shown in Fig. 5. Figure 5 shows that the synchronization performance of the proposed method could be remarkably improved by increasing the differential path number $N$. The synchronization performance 

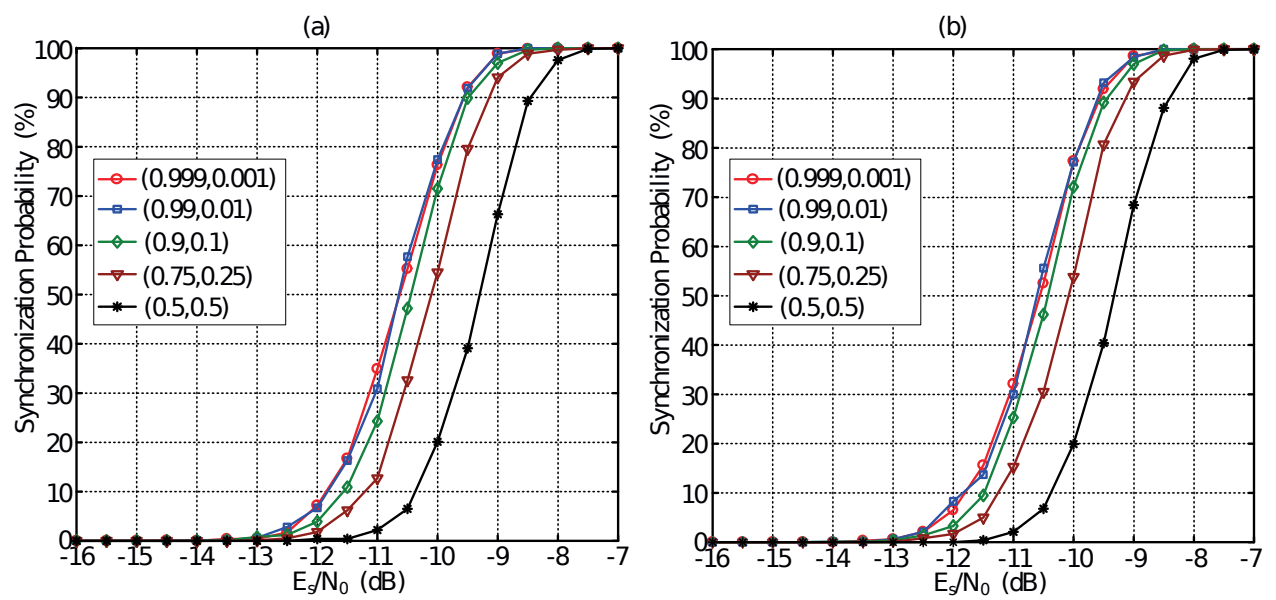

Fig. 4. Synchronization probabilities of different weighting factors, differential path number $N$ of 8 and AWGN channel: (a) FO value of $200 \mathrm{kHz}$, (b) FO value of $4 \mathrm{MHz}$.

(a)

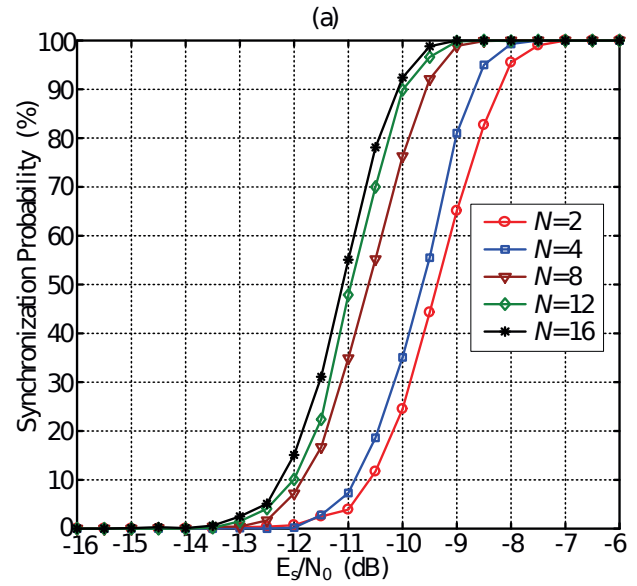

(b)

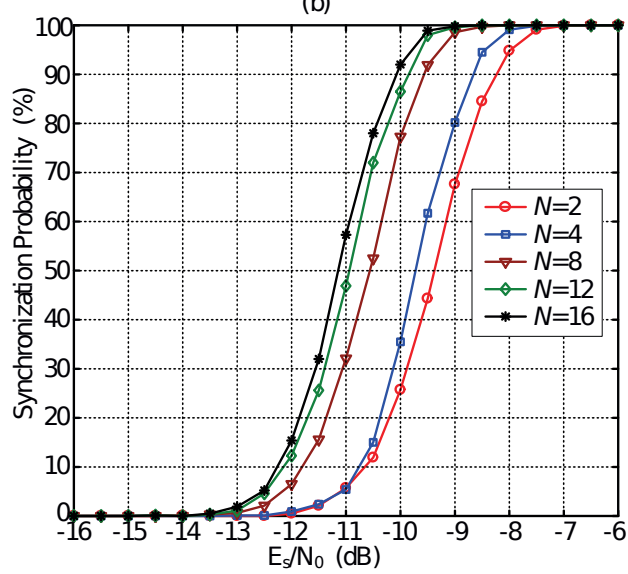

Fig. 5. Simulation results of different path numbers, $\mu_{1}=0.99, \mu_{2}=0.01$ and AWGN channel: (a) FO of $200 \mathrm{kHz}$, (b) FO of $4 \mathrm{MHz}$.

is enhanced by approximately $1 \mathrm{~dB}$ by increasing $N$ from 4 to 8 , but the performance increment would keep decreasing along with the path number $N$, for example, the improvement of approximately $0.4 \mathrm{~dB}$ for $N$ ranging from 8 to 12 and the improvement of less than $0.2 \mathrm{~dB}$ for $N$ ranging from 12 to 16 . Consequently, the differential path number needs to be set to 8 for practical applications. ${ }^{1}$

\section{Conlusion}

In this paper, we proposed a synchronization method based on combining multi-differential correlations for the satellite communication link with high Doppler. We investigated the proposed method in detail for the resisting FO and noise. The mathematical closed expressions were derived, such as the maximum acceptable FO, synchronization probability, and pure correlation PNR. The theoretical analysis and simulation results show that the proposed method has a strong ability to resist the FO, and its performance is almost steady for different FOs. As compared with the CDCS method, the minimum $E_{\mathrm{s}} / N_{0}$ of the proposed MDCS method corre- sponding to the synchronization probability of $99 \%$ can be decreased by approximately $6 \mathrm{~dB}$ for a small $\mathrm{FO}$ and by more than $3 \mathrm{~dB}$ for a large FO. In general, the proposed method can enhance the capability of rejecting the FO as compared with NDCS method and the anti-noise property in contrast with CDCS method. In the future, we will study the application of proposed method in broadband communication.

\section{Acknowledgments}

This project is supported by supported by the National Natural Science Foundation of China under Grant 62001438 and the Postdoctoral Fund of Hebei Province under Grant B2019003033.

\footnotetext{
${ }^{1}$ The URL of the detailed description of MATLAB simulation is https://pan.baidu.com/s/1PRWccz2KyKxYbmktqtkWGw.
} 


\section{References}

[1] KOZLOWSKI, S. A carrier synchronization algorithm for SDR-based communication with LEO satellites. Radioengineering, 2018, vol. 27 , no. 1, p. 299-306. DOI: 10.13164/RE.2018.0299

[2] LV, H. C., LU, X. C., WU, J. F. A method of two-way satellite-ground time synchronization under inter-satellite links system. Yuhang Xиebao/Journal of Astronautics, 2017, vol. 38, no. 7, p. 728-734. DOI: 10.3873/j.issn.1000-1328.2017.07.008

[3] WANG, L, XU, D. An anti-frequency offset fine time synchronization method and its performance analysis. Journal of Electronics \& Information Technology, 2011, vol. 33, no. 2, p. 300-303. DOI: 10.3724/SP.J.1146.2010.00346

[4] MA, Y., ZHOU, S., YAN, C., et al. Design of OFDM timing synchronization based on correlations of preamble symbol. In Proceedings of the IEEE 83th Vehicular Technology Conference(VTC Spring). Nanjing (China), 2016, p. 1-5. DOI: 10.1109/VTCSpring.2016.7504190

[5] GUL, M. M. U., MA, X., LEE, S. Timing and frequency synchronization for OFDM downlink transmissions using Zadoff-Chu sequences. IEEE Transactions on Wireless Communications, 2015, vol. 14, no. 3, p. 1716-1729. DOI: 10.1109/TWC.2014.2372757

[6] Al-HADDAD, M. K., ZIBOON, H. T. Joint carrier frequency and symbol timing synchronization sequence for FBMC based system. Physical Communication, 2020, vol. 42. DOI: $10.1016 /$ j.phycom.2020.101165

[7] DAS, A., MOHANTY, B., SAHU, B. Modified CAZAC sequence based timing synchronization scheme for OFDM system. Wireless Personal Communications, 2019, vol. 108, no. 1, p. 37-49. DOI: $10.1007 / \mathrm{s} 11277-019-06386-y$

[8] JIANG, Y. WANG, Y. CAO, P., et al. Robust and low-complexity timing synchronization for DCO-OFDM LiFi systems. IEEE Journal on Selected Areas in Communications, 2018, vol. 36, no. 1, p. 53-65. DOI: 10.1109/JSAC.2017.2774419

[9] LI, L., YAO, W., HAN, L., et al. Timing synchronization algorithm based on FH sequence for coherent optical OFDM systems with carrier frequency offset. Optoelectronics Letters, 2019, vol. 15, no. 4, p. 288-291. DOI: $10.1007 / \mathrm{s} 11801-019-8171-9$

[10] MAREY, M., STEENDAM, H. Analysis of the narrowband interference effect on OFDM timing synchronization. IEEE Transactions on Signal Processing, 2007, vol. 55, no. 9, p. 4558-4566. DOI: 10.1109/TSP.2007.896020

[11] CUI, G., HE, Y., LI, P., et al. Enhanced timing advanced estimation with symmetric Zadoff-Chu sequences for satellite systems. IEEE Communications Letters, 2015, vol. 19, no. 5, p. 747-750. DOI: 10.1109/LCOMM.2015.2411610

[12] LI, P., HE, Y., CUI, G., et al. A novel timing advanced estimation algorithm for eliminating frequency offset in satellite system. In 2015 IEEE 26th Annual International Symposium on Personal, Indoor, and Mobile Radio Communications (PIMRC). Hong Kong (China), 2015, p. 1792-1796. DOI: 10.1109/PIMRC.2015.7343589

[13] ZHAO, Y., CAO, J., LI, Y. An improved timing synchronization method for eliminating large Doppler shift in LEO satellite system. In Proceedings of the IEEE 18th International Conference on Communication Technology (ICCT). Chongqing (China), 2018, p. 762-766. DOI: 10.1109/ICCT.2018.8600170
[14] JIE, T., FANG, W., MINGQI, L. Synchronization based on preamble symbol for DVB-T2 system. Tongxin Xuebao/Journal on Communications, 2017, vol. 38, no. 8, p. 1-7. DOI: 10.11959/j.issn.1000-436x.2017167

[15] ZARRABIZADEH, M., SOUSA, E. A differentially coherent PN code acquisition receiver for CDMA systems. IEEE Transactions on Communications, 1997, vol. 45, no. 11, p. 1456-1465. DOI: $10.1109 / 26.649772$

[16] VILLANTI, M., SALMI, P., CORAZZA G. E. Differential post detection integration techniques for robust code acquisition. IEEE Transactions on Communications, 2007, vol. 55, no. 11, p. 2172-2184. DOI: 10.1109/TCOMM.2007.908535

[17] REY IGLESIAS, D., GARCIA SANCHEZ, M. Semi-Markov model for low elevation satellite-earth radio propagation channel. IEEE Transactions on Antennas and Propagation, 2012, vol. 60, no. 5, p. 2481-2490. DOI: 10.1109/TAP.2012.2189707

[18] LOPEZ-SALCEDO, J. A. Simple closed-form approximation to Ricean sum distributions. IEEE Signal Processing Letters, 2009, vol. 16, no. 3, p. 153-155. DOI: 10.1109/LSP.2008.2012223

\section{About the Authors...}

Jian-cheng LIU (corresponding author) was born in Handan, China in July 1987. He received his B.E. degree and M.E. degree from Electronic Engineering Institute of PLA respectively in 2010 and 2013. He received his Ph.D. degree of Control Science and Engineering from Mechanical Engineering College, China, in 2016. He is now a postdoc at the 54th Research Institute of China Electronics Technology Group Corporation. His research interests include antijamming technology of satellite communication and adaptive communication signal processing.

Sai-yu WANG was born in Neimenggu, China in 1976. He received his M.E. degree from Nankai University, China in 2002. Now he is a reseacher in the 54th Research Institute of China Electronics Technology Group Corporation. His research interest is anti-jamming technology of satellite communication.

Zhi-song HAO was born in Baoding, China in 1978. He received his M.E. degree from the 54th Research Institute of China Electronics Technology Group Corporation in 2005. Now he is a reseacher in the 54th Research Institute of China Electronics Technology Group Corporation, and his research interest is satellite communication.

Jing-tao ZHANG was born in Shijiazhuang, China in 1989. He received his B.E. degree from Shanghai Jiao Tong University, China in 2011, and now is a Ph.D. student at the 54th Research Institute of China Electronics Technology Group Corporation. 